\title{
10. Thinking beyond means-ends analysis: the role of impulse-driven human creativity in the design of artificially intelligent systems
}

\author{
DONGMING XU \\ YONGGUI WANG \\ SUKANTO BHATTACHARYA \\ UNIVERSITY OF QUEENSLAND
}

\section{Abstract}

While goal-directed problem solving as advocated by Herbert Simon's meansends analysis model has primarily shaped the course of design research on artificially intelligent systems, we contend that there is a definite disregard of a key phase within the overall design process that in fact logically precedes the problem-solving phase. While the systems designers have been obsessed with goal-directed problem solving, the basic determinants of the desired goal state remain to be fully understood or categorically defined. We propose an argumentative framework built on a set of logically interlinked conjectures that seeks to specifically highlight the importance of this hitherto neglected phase in the overall design process of intelligent systems.

\section{Introduction}

There are at least two distinct forms of human creativity that motivate design research: one that is primarily goal driven and is essentially concerned with 'problem solving'; and one that is impulse driven and is essentially concerned with 'problem creation' (Michalos 1970). Michalos opines that while there could be some common factors that underlie both forms, there are critical cognitive distinctions in terms of the intellectual as well as emotional drivers that are 
involved in problem-creating as opposed to problem-solving design research. Herbert Simon's (1969) initial attempt at collating the creative activity engaged in by 'every liberally educated man' (including scientists, artists and engineers), by exhorting them to share their professional experiences thus enhancing the cumulative outcome of the creative design process, stopped short of fully recognising these distinctions. So we contend that Simon's means-ends analysis (MEA) framework can be appropriately applied only to design research that has a definite problem-solving goal.

In this context, the design activity of 'problem creation' that we refer to is entirely separate from the task of 'problem recognition', which usually forms the beginning step in a problem-solving design activity. The outcome of a 'problem-creation' activity is a concept or idea (and not necessarily a thing) that has the characteristic of 'absolute newness' — a term we shall encounter and elucidate later on.

Design research in a few fields (including information and knowledge engineering) is synonymous with improvement research - a designation that evidently emphasises the goal-driven nature of the involved problem-solving and/or performance-enhancing activities. This is perhaps attributable to a rather mechanistic view of systems design with origins in some of the early artificial intelligence (AI) programs of Simon - for example, the General Problem Solver (GPS) developed in 1957, which was simply an algorithmic execution of the MEA model for heuristic problem solving (Frantz 2003).

In this chapter, we will first critically examine the role played by the MEA framework in shaping the course of artificially intelligent systems (AIS) design over its fairly recent history and then go on to propose a systematic framework to help understand the equally vital (but hitherto largely neglected) role of impulse-driven creativity and resulting implications.

\section{Influence of MEA on AIS design}

When Newell and Simon first programmed the GPS (Newell et al. 1959), there was no clear distinguishing line, so to speak, between digital computer and machine intelligence. In fact, Simon posited his science of the artificial with digital computer being the artefact of interest. So the design of the GPS could have been stimulated partly by parallel developments in other design sciencesespecially in mechanical and construction engineering, which were looking for increasingly efficient ways to numerically solve complex applied mathematical problems pertaining to those fields. While GPS was the first formal computer program that successfully separated the domain of 'problem knowledge' from that of 'solution strategy', there was, however, very little 'problem-creation' 
activity involved in designing GPS, as well as later variants such as the STRIPS (Stanford Research Institute Problem Solver). This was quite obvious given that these programs were nothing more than algorithmic implementations of the classical MEA model whereby an existing problem was first visualised in the form of a 'current' (that is, problem) state and a 'goal' (that is, solution) state. Once any given problem has been so visualised, the heuristic solution technique proceeds by choosing an action from a set of available alternatives (often mutually exclusive) so as to reduce the gap between the two states. An action leads to a new (often intermediate) state, which is somewhat better than the original state but somewhat worse than the desired goal state. This new state then becomes the current state and the process is applied recursively until the features of the current and the goal states become virtually indistinguishable (Fikes and Nilsson 1971).

An important aspect of goal-directed problem solving as applied in MEAbased intelligent systems such as GPS and STRIPS is a logical (often sequential) framework in which actions need to be taken in order to attain a particular goal (Simon 1996). Such a goal-directed problem-solving system is required to be connected to the external environment by sensory connectivities through which it can collate environmental information and motor connectivities through which it can transmit the collated information to some kind of a central processor and then react to the external stimuli in accordance with the outcome of such information processing. In addition, such a system has to have some means of storing pre-processed as well as post-processed information-that is, some sort of internal memory (Stuart and Norvig 2003). In other words, such a system is required not only to emulate the behaviour of the human designer, but in order for it to be able to do so, it has to have a rather similar internal physical organisation. This had led to a belief among a number of pioneering AI researchers that both the power and the performance of intelligent systems could be improved without bounds by simply improving their structural design so as to continually approximate the internal structure of more and more complex biological organisms, ultimately creating an 'artificial human' that could think, behave and communicate exactly like a real person. So AIS design researchers have been largely engrossed within a utopian comfort zone, believing that biological evolution has already done the hard work for them via the Darwinian process of natural selection in coming up with what is the most optimal design for an intelligent system, and all they need to do is simply follow. So, creativity in the context of machine intelligence has become nearly synonymous with targeted problem solving, thereby limiting intelligent system design research to the designing of artefacts that are more effective in physically emulating natural intelligence. 
Such a general belief among pioneering AIS designers was clearly evident in the burgeoning of interest in the design of expert systems that were 'taught' via a supervised learning process to gain expert knowledge in a particular problem domain and then take decisions or render advice much like a human expert. Dendral is a well-known case of such an expert system whose main goal was to assist chemists to identify unknown organic molecules by analysing their mass spectra and utilising a chemical knowledge base (Lindsay et al. 1980). As an example of emulating human thinking within a less sombre but nevertheless problem-solving domain, chess-playing computer programs have been designed with the goal of first learning from and then beating their human masters at their own game (Levy and Newborn 1991). A parallel growth has also been observed, alongside human behaviour, in the design of AIS that attempt better emulation of human communication by means of natural language processing. An example of one of the earliest of such an expert system is Siklossy's ZBIE program (Siklossy 1972).

What about creativity? Can creativity be artificially generated? This is a really tough question. Never-say-die AI researchers have tended to tackle the problem of creativity by essentially transforming the question itself so that answers can be sought within some sort of MEA framework. To that effect, software programs have been written that it is claimed are 'creative' to the extent that they have demonstrated a rudimentary capability in replicating some form of creative human behaviour - for example, being able to compose a story, such as MINSTREL (Turner 1994), or paint a picture, such as 'AARON' (McCorduck 1991). The pertinent question, however, is: can one say that a piece of software is creative just by observing its output in the same sense that one can say a human child is creative just by observing the behaviour of the child at play? The fact is that while much of primordial human creativity is impulse driven, that of machine-generated creativity is goal driven and, while sometimes the distinguishing line between the two can appear very thin, that line can never completely fade out.

\section{Where the problem lies}

While the goal of natural-science research is truth, that of design-science research is utility. Pursuits of truth and utility are, however, ontologically inseparable since one informs the other-'an artefact may have utility because of some yet undiscovered truth while a theory may yet to be developed to the point where its truth can be incorporated into design' (Hevner et al. 2004). For example, the initial designs of flying machines were inspired largely by simply observing the flight of birds, but those rudimentary designs did make a contribution towards the subsequent birth and enrichment of the theory underlying modern aviation science. And although modern aircraft operate very differently to how birds 
fly, as Brooks points out, they did not lose their utility as an artefact even when the governing design deviated over time from the biological inspiration that motivated the original (Hearst and Hirsh 2000).

According to Aaron Sloman, any intelligent systems design process has two guiding themes: science, which is concerned with investigating natural intelligence and what alternative types of intelligence are possible and how they might be artificially embodied, and engineering, which is concerned with the building of useful things (Hearst and Hirsh 2000).

While the engineering theme of intelligent systems design has to do with problem solving-type tasks principally associated with the MEA framework of design science (the part of the flow chart that is enclosed within a dashed box in Figure 10.1), the science theme has to do more with the impulse-driven creativity aspect of design science since it is concerned with problem-creation and problem-identification tasks as distinct from problem solving.

So, while the engineering theme deals with the 'how' questions, the science theme has to tackle the 'why' ones. While it is obvious that both the 'why' and the 'how' questions need to be systematically answered in the overall design process to ensure a fruitful outcome, there is an implied natural order of precedence: the 'why' questions need to be tackled before the 'how' ones.

The successful dovetailing of aviation science into aeronautical engineering shows that only after a problem has been properly 'created' by adequately answering a basic 'why' question can the science theme of design meaningfully give way to the engineering one, which subsequently tries to answer all the ensuing 'how' questions of design.

What, however, has happened rather well in the case of flying-machine design has not happened quite so successfully in the case of intelligent machine design. The primary reason for this has been the ineffective dovetailing of information science into systems engineering. Historically, designers of AIS have tended to be more concerned with answering the 'how' questions first and have not bothered too much with the 'why' one - as a result of which the temporal growth path of AIS design has become somewhat lopsided. As Donald Mitchie has very correctly noted, there is a consistent disregard of Alan Turing's (1950) classical paper on AI in which a two-stage approach was proposed: first, to construct a teachable machine, and second, to subject the constructed machine to a course of education. As a result of this disregard towards a classical study that hinted at a basic 'why' question, the design process of intelligent machines in general and AIS in particular has become back-to-front: researchers are trying to improve the system design for obtaining solutions to a problem that still remains to be adequately created and understood (Hearst and Hirsh 2000). So, while not 
denying the importance of the problem-solving approach based on MEA in initiating research on AIS design, it has now become quite necessary to also explore the crucial role that impulse-driven creativity has to play in this matter.

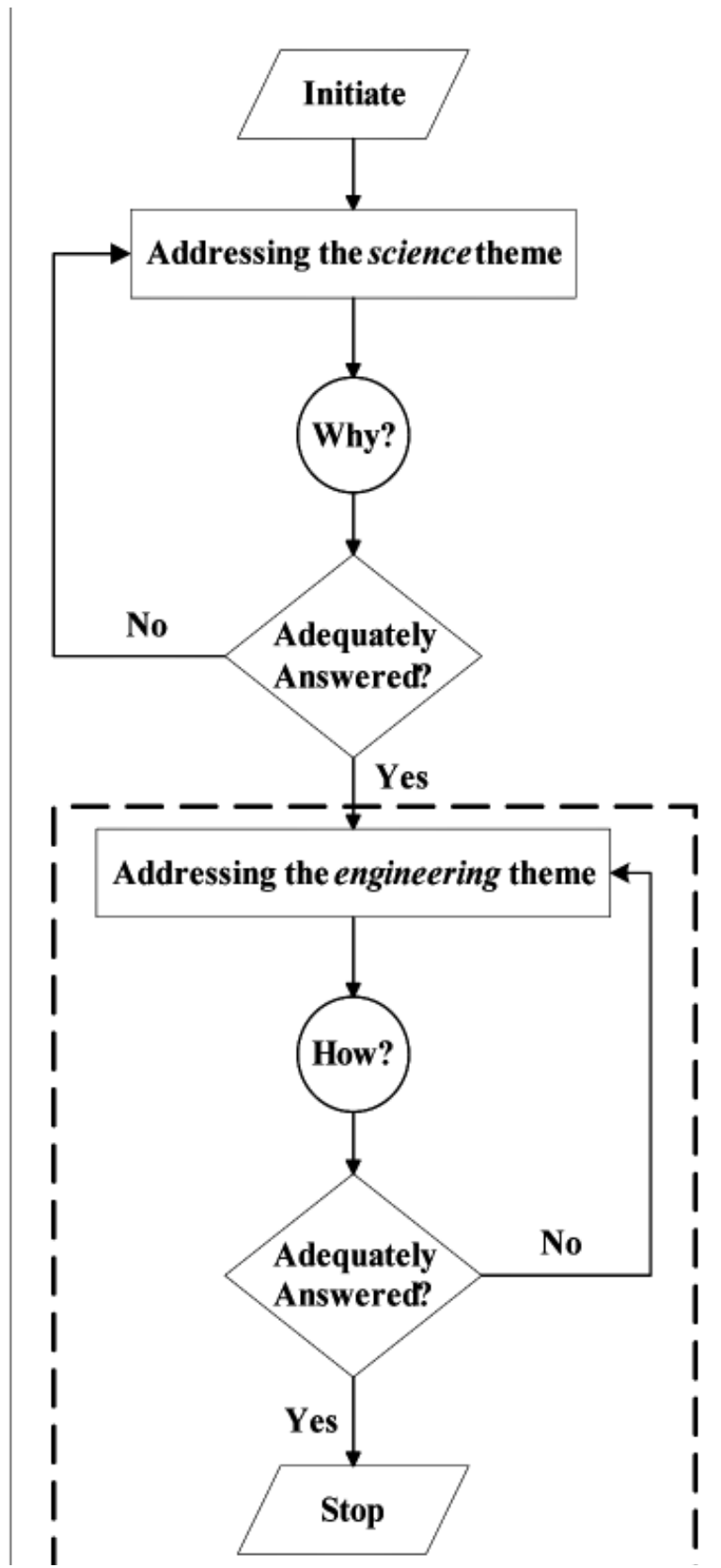

Figure 10.1 Flow chart depicting a sequential ordering of Sloman's suggested intelligent systems design themes 


\section{A systematic framework for highlighting the role of impulse-driven creativity}

Subrata Dasgupta (1992) posits two qualitative laws, which he claims are valid throughout any science of design. He argues that since a major aim of design theory is 'to construct explanatory models that further enhance our understanding of design as a cognitive act', there ought to be certain fundamental laws of design that are valid across all the sciences of the artificial just as there are certain fundamental physical laws that always hold true across all the natural sciences.

The two design laws proposed by Dasgupta are: the Hypothesis Law, which states that 'a design process that reaches termination does so through one or more cycles of hypothesis creation, testing and (if necessary) modification'; and the Impermanence Law, which states that 'a design in any given state is never guaranteed to remain in that state'. While the first of Dasgupta's laws applies to design as a systematic process, the second applies to design as an outcome of such a systematic process. Even without going into any extended debate concerning the validity of these laws, it is not too difficult to see that these laws apply exclusively to design motivated by creativity that is primarily goal driven and concerns a problem-solving activity. In coming up with his design laws, Dasgupta draws heavily from two of the pioneers of the MEA framework as applied to AIS design: Newell's (1982) knowledge-level paradigm and Simon's (1979) bounded rationality. Dasgupta, however-possibly for the first time - presents a formal argument in support of 'newness' being an essential prerequisite for a design process to be initiated. He, however, constrains his definition of 'newness' so as to agree with MEA by considering it in relative terms: newness is considered relative to the designer's knowledge. This essentially brings the concept as defined by Dasgupta within the bounds of the dashed box in Figure 10.1.

It has also been argued that the creativity behind some original design inspirations can be explained via a mechanism of 'analogical transfer' (Bhatta and Goel 1997). The concept of 'absolute newness' that we touched on in the introductory section is not, however, fully explained by this line of argument, as many significant inventions in human history have had very few if any clear analogical connections with a pre-existing artefact. Indeed, in many instances, analogical connections were drawn only after a rudimentary design of the artefact of interest had already been conceived through an act of purely impulsedriven creativity. In an apparent realisation of the uniqueness of impulse-driven human creativity, Dasgupta (2008) has recently posited that 'computational models of the creative process are fundamentally flawed as theories of human 
creativity' and the power of computational models lies elsewhere. We contend that 'absolute newness' - as the hallmark of impulse-driven creativity - must possess two essential characteristics.

- To be considered 'absolutely new', the design of an artefact should not draw from any a priori body of knowledge or repository of ideas. For example, although there is no historical evidence to suggest that he in fact tried to physically build a flying machine (Gray 2006), Leonardo da Vinci's fifteenthcentury drawings did initiate a design process with the flying machine as the artefact of interest with no known a priori body of scientific knowledge to inspire or guide his drawings of such a machine. Following Boden (1990), creativity associated with 'absolute newness' does not arise from explorations restrained within the boundaries of a pre-specified conceptual space, rather 'from a deliberate transformation or transcendence of this space'. This also corresponds to 'H-creativity' (creativity recognised as novel by the society at large) rather than 'P-creativity' (novel only to the agent producing it) (Boden 1999).

- 'Absolute newness' is not to be associated with innovation. Although they are sometimes used interchangeably, 'creativity' and 'innovation' are two distinct concepts. Innovation in design results from the successful implementation of a created concept or idea (Cooper et al. 1995). While all innovation involves some creativity, not all creativity, however, is innovative. In that sense, da Vinci was certainly a creator but not an innovator. The Wright brothers, however, were innovative to the extent that they in fact tried to give a physical form to their creativity. Subsequent aircraft designers were mostly innovators who primarily engaged in problem solving to improve on the existing states of design (in line with Dasgupta's second law), exhibiting goal-driven creativity.

Once a problem has been adequately created, it can be tackled effectively so that a solution can be found; a desired goal state can be achieved. Goal-driven creativity then takes over from impulse-driven creativity as acts of innovation start to improve an 'absolutely new' artefact.

So, collating the concepts discussed, we state our Conjecture 10.1 as follows.

\section{Conjecture 10.1}

As the nature of the primary activity constituting a design process shifts from problem creation towards problem resolution, the form of human creativity motivating such a process shifts from being impulse driven towards being goal driven.

This is depicted in Figure 10.2, building on the flow chart in Figure 10.1. 


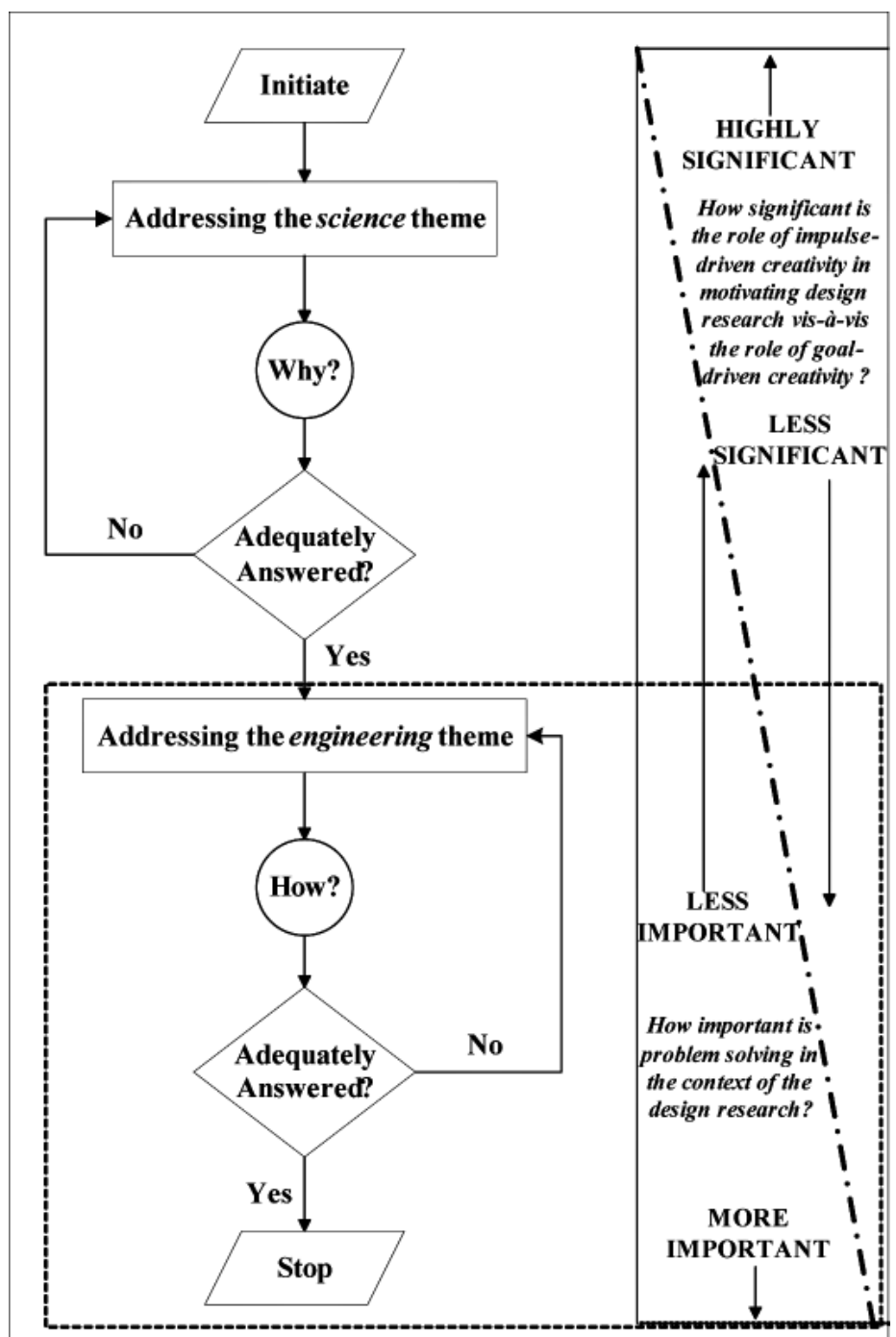

Figure 10.2 Flow chart depicting the relation between forms of creativity and the associated design activities

So goal-driven creativity should play an increasingly significant role as the design process begins to address the 'how' questions. This conceptualisation is fairly intuitive given that much of the engineering theme of a design process is concerned with improvement research - that is, how an artefact of interest should ideally shape up. In other words, it is concerned with attaining a more and more refined state of design. Defining logically precedes refining, however, and this is recognised even in the MEA framework itself in that it does put 'problem recognition' as the initiating step. Within the MEA framework, 
however, problem recognition is indistinguishable from goal setting. Problem creation is, however, something quite different; it initiates the design process underlying the science theme as shown in Figures 10.1 and 10.2.

Any artificially intelligent system - at its most refined state of design - would be expected to pass the Turing test (or a comparable testing process) on a global scale, not just within the constraining bounds of a specific problem domain (Turing 1950). Although ELIZA and PARRY are two of the earliest rule-based programs that were acclaimed to have 'passed' this test (Weizenbaum 1966; Colby 1975), it has since been argued that such rule-based systems have been able to fool a human examiner into mistaking them for human only within a rather restricted problem domain.

Moreover, it has been argued that passing the Turing test is a necessary but not a sufficient proof of a machine's ability to think (Searle 1980). So, even if one remains entirely within the MEA framework, it is still not very clear what is the ultimate goal state of AIS design since the determinants of that state are still disputed. The early designers of flying machines did thoroughly study and were able to broadly understand the physiological mechanisms that enabled birds to fly, identifying the exact mechanisms that were lacking in human beings making them incapable of flight. They answered the 'why' question and thereby determined the basic determinants of the desired goal state. Pioneers of intelligent systems design have, however, neglected the need to first understand the mechanisms that determine intelligent thought - to satisfactorily answer a very basic 'why' question: 'why cannot machines think like human beings?'

Thus, AIS designers do not know and cannot say at this time what should ideally replace the question mark in Figure 10.1; is it human thought (mind) or human brain (matter)? The current AIS design state does not have a unique answer.

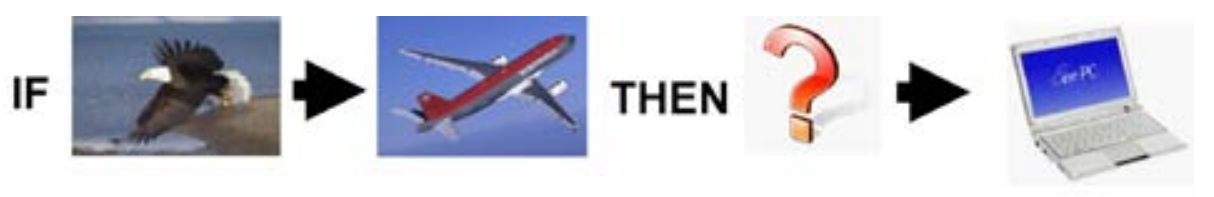

Figure 10.3 A conceptual representation of the missing 'why' in AIS design

So, again collating the concepts discussed, we state our Conjecture 10.2 as follows.

\section{Conjecture 10.2}

All the determinants of a desired goal state have to be fully understood and categorically defined before a design process can be productively motivated by human creativity of a purely goal-driven form. 
This is depicted in Figure 10.4, building on the flow chart in Figure 10.2.

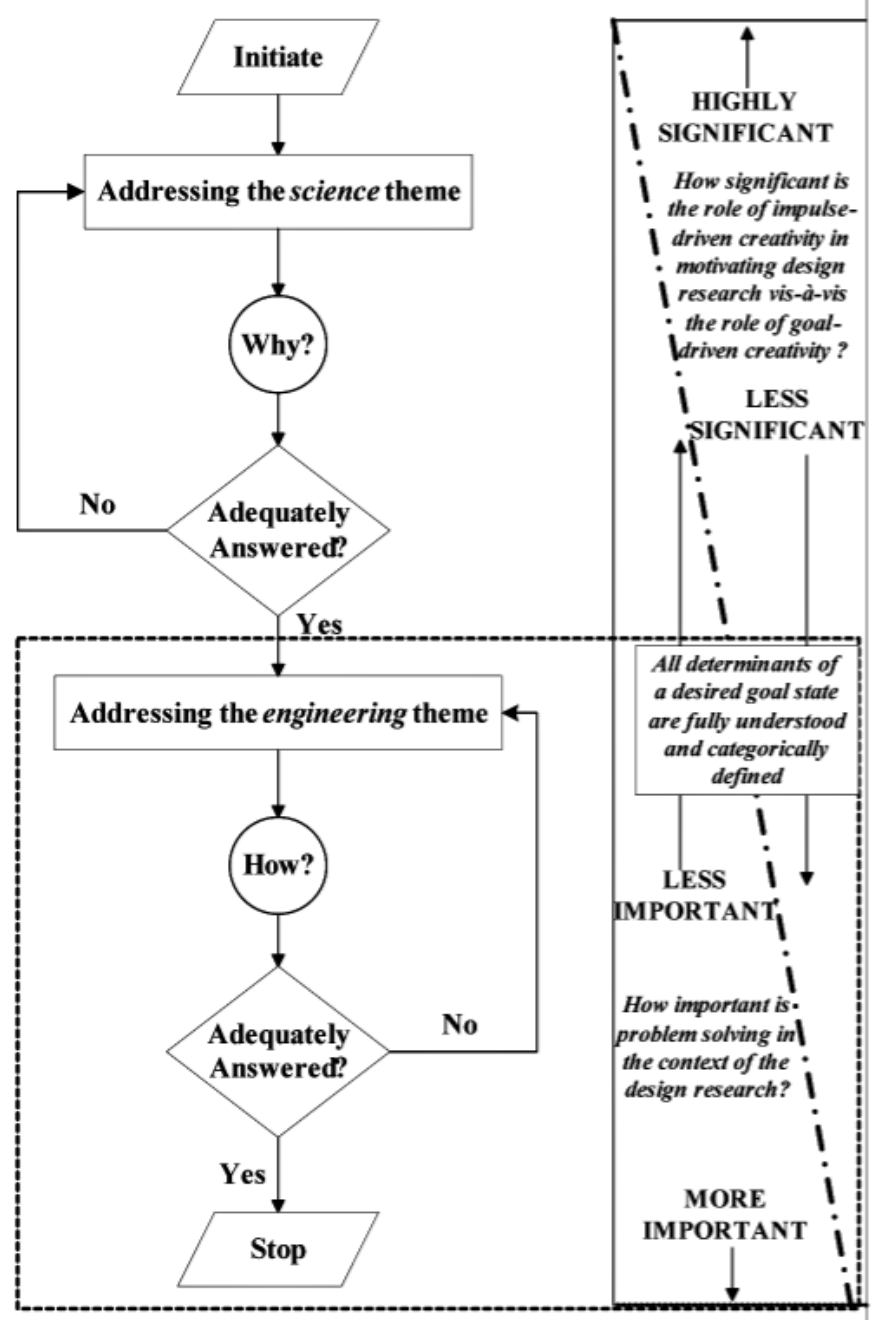

Figure 10.4 Flow chart depicting the need for understanding and defining all goalstate determinants before problem-solving design research can productively ensue

Our third conjecture arises out of the intersection of Conjectures 10.1 and 10.2 and is stated as follows.

\section{Conjecture 10.3: $\rightarrow\{\mathrm{C} 1 \cap \mathrm{C} 2\}$}

For a design process to progress productively towards successful culmination, the real shift of the form driving human creativity from impulse driven to goal driven must necessarily occur only after the determinants of a desired goal state have been completely understood and categorically defined.

This is depicted in Figure 10.5, building on the flow chart in Figure 10.4. 


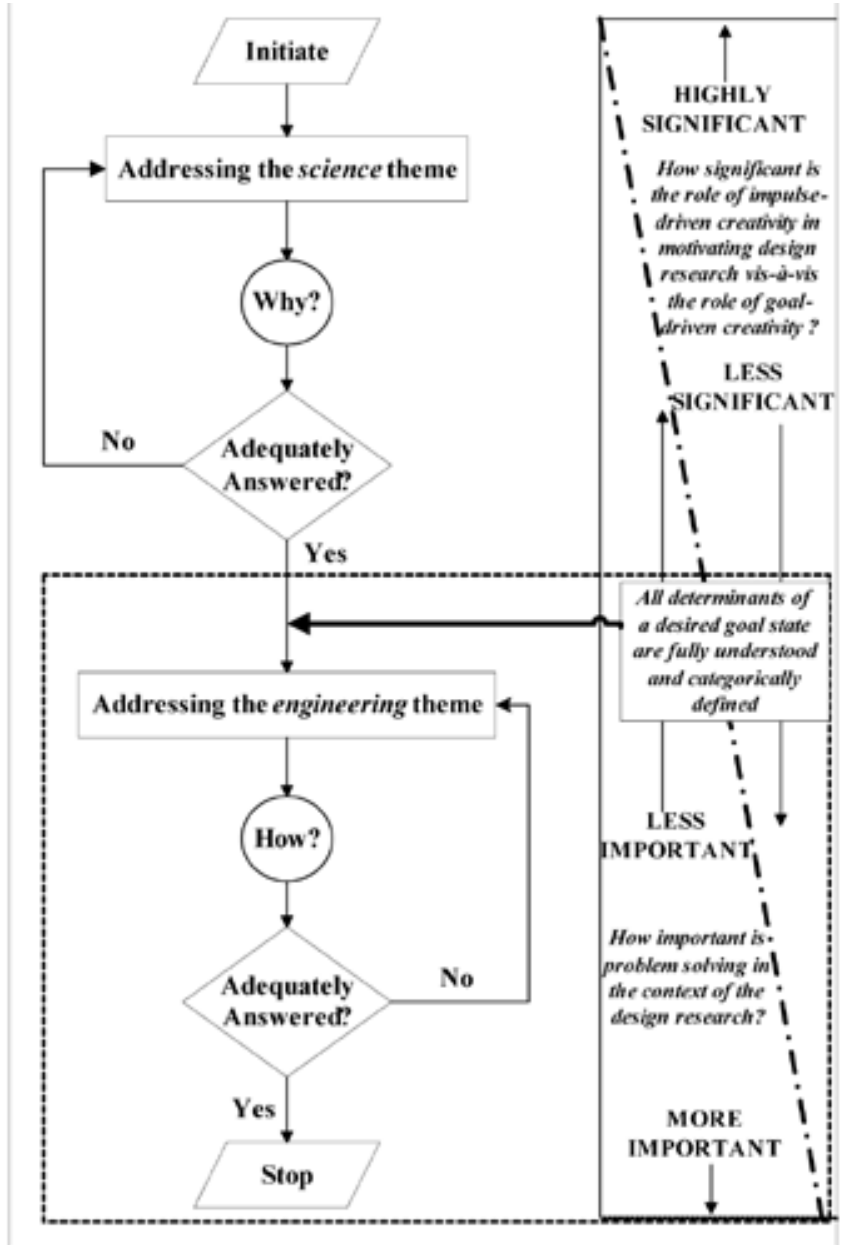

Figure 10.5 Flow chart depicting the precedence of understanding and defining determinants of the goal state over the onset of the problem-solving phase by goaldriven creativity

Given that the desired goal state of the intelligent system design process is artificial production of intelligent thought, until and unless all the determinants of intelligent thought are thoroughly understood, such a design process is very unlikely to culminate in the desired goal state if it is motivated purely by goaldriven human creativity. Thus, we state Conjecture 10.4 as follows.

\section{Conjecture 10.4}

Only after the 'why' question relating to the science theme of the design process is adequately resolved can all the determinants of a desired goal state be completely understood and categorically defined.

This is depicted in Figure 10.6, building on the flow chart in Figure 10.5. 


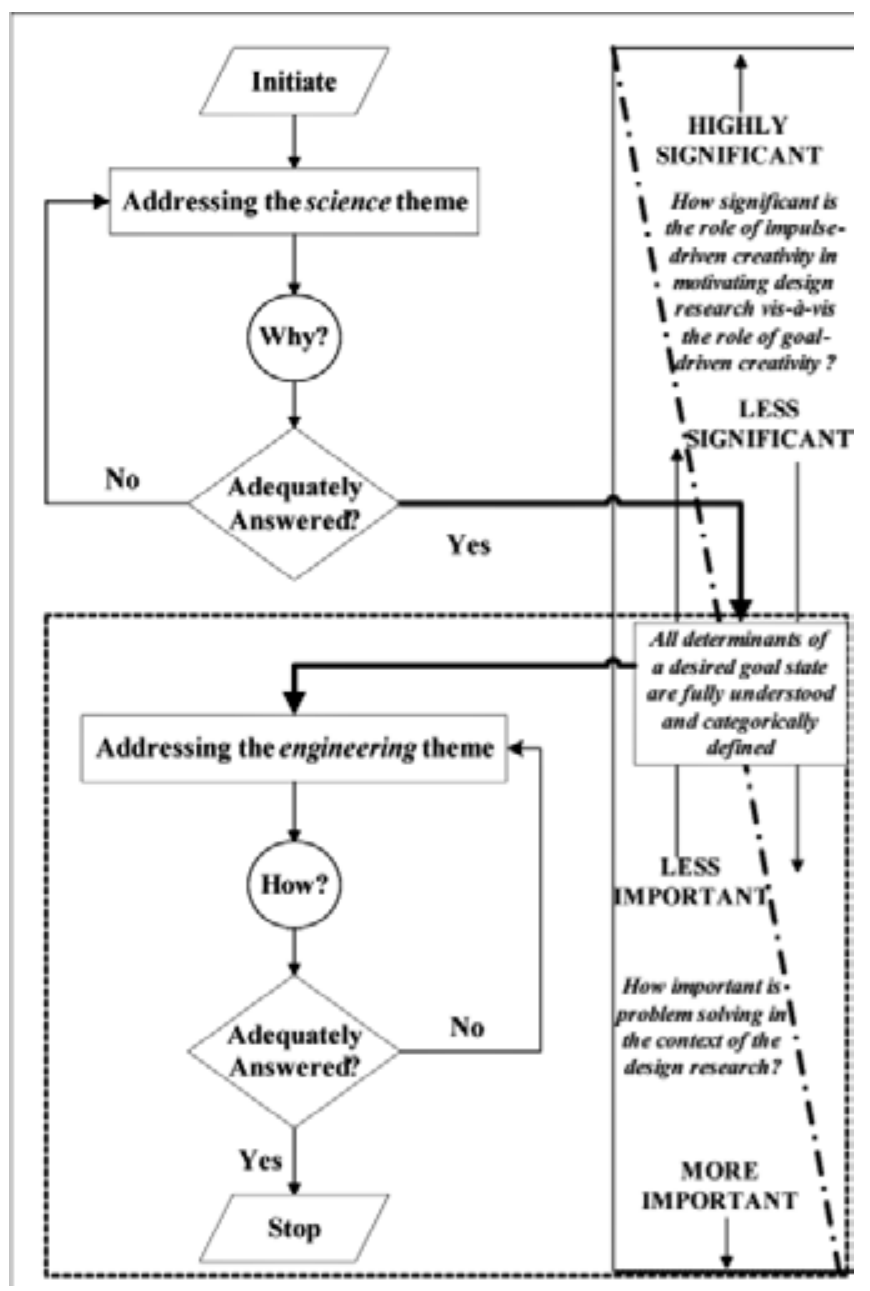

Figure 10.6 Flow chart depicting that the successful tackling of the 'why' question allows the determinants of the goal state to be fully understood and categorically defined

As we have stated previously, however, all innovation involves creativity but not all creativity is innovative. Innovation is indispensable for a design process to culminate successfully, to attain the desired goal state. It is essential to innovate in order to transform a creative idea into an artefact of interest. So, innovation might essentially be what jump-starts the problem-solving phase of a design process by bringing about some sort of practical implementation of the creative idea on which later designers can then improve. This brings us to our Conjecture 10.5, which we state as follows. 


\section{Conjecture 10.5}

Occurrence of innovation triggers the end of the problem-creating phase and the onset of the problem-solving phase in the overall design process.

This is depicted in Figure 10.7, building on the flow chart in Figure 10.6.

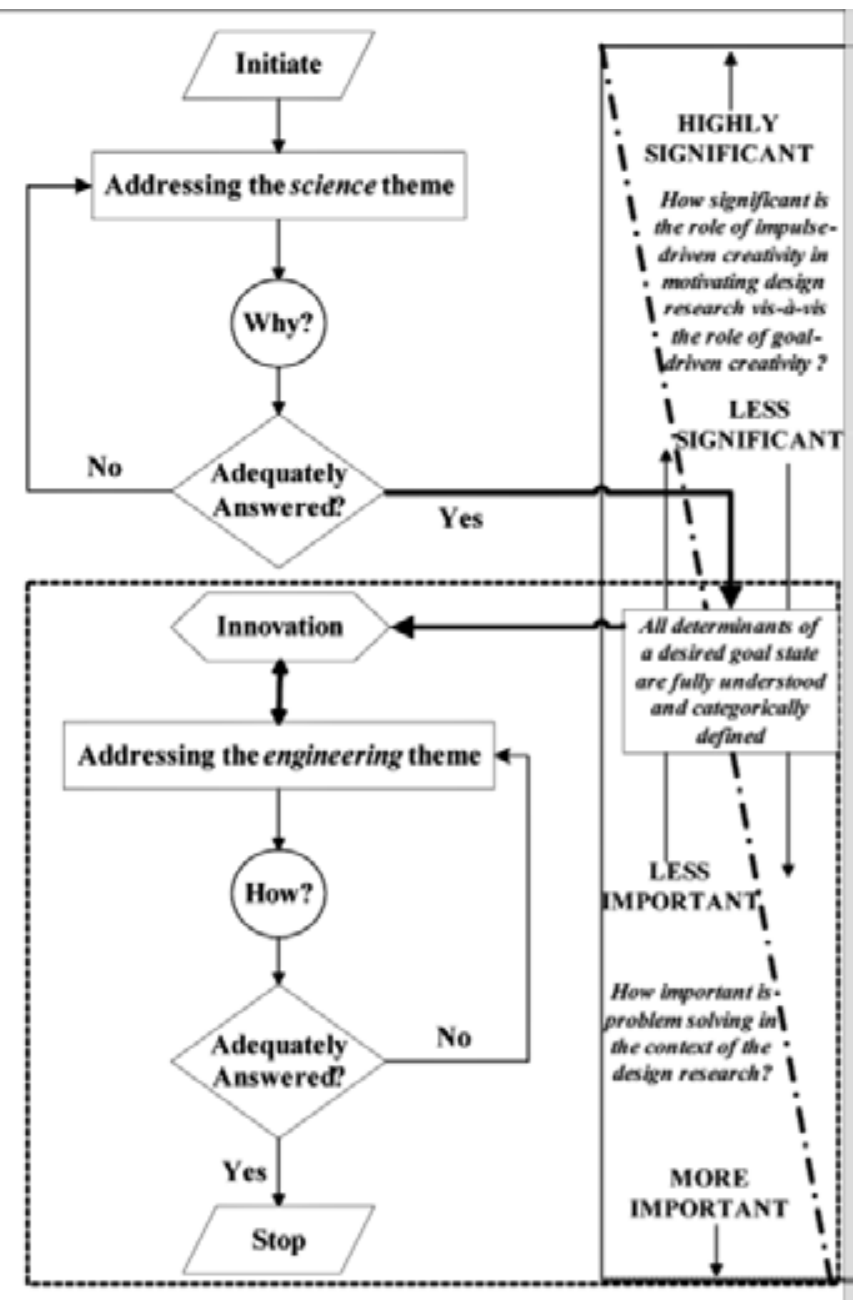

Figure 10.7 Flow chart depicting the onset of innovation as a trigger for the start of the problem-solving phase and the end of the problem-creation phase

The first time innovation occurs in a design process, it effectively prepares the ground for the engineering theme to be addressed. The double-headed arrow in Figure 10.7 indicates, however, that the process of addressing the engineering 
theme can also contribute to subsequent innovation (that is, re-innovation) as long as a current design state can be improved on and as long as all issues contributing to the 'how' bit have not been adequately addressed.

Our sixth conjecture arises out of the intersection of Conjectures 10.4 and 10.5 and is stated as follows.

\section{Conjecture 10.6: $\rightarrow\{\mathrm{C} 4 \cap \mathrm{C} 5\}$}

Innovation can start to effectively occur only after the 'why' question of the science theme of design has been adequately answered.

This is depicted in Figure 10.8, building on the flow chart in Figure 10.7.

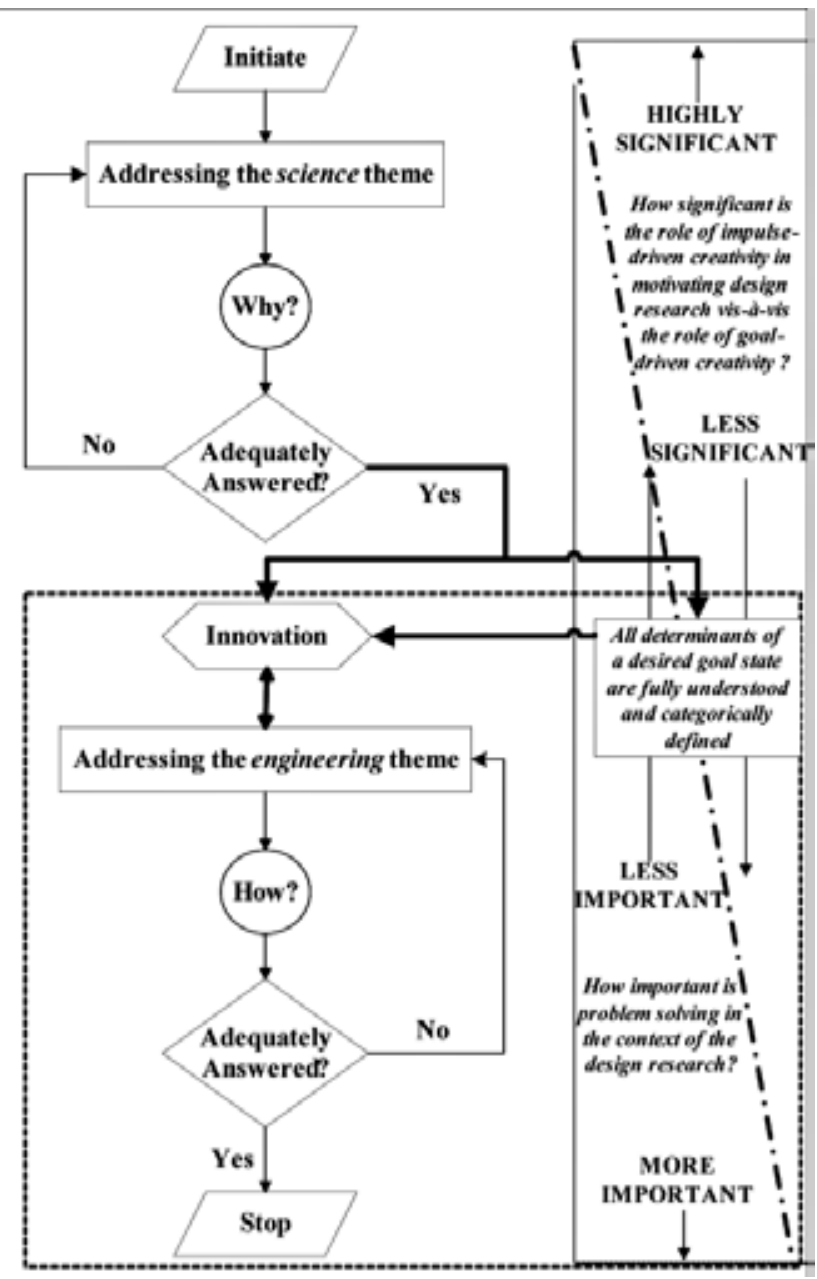

Figure 10.8 Flow chart depicting the precedence of answering the 'why' question pertaining to the science theme over the effective onset of innovation 
Combining our six conjectures, our final and all-enveloping conjecture is a union of Conjectures 10.3 and 10.6 and is thus stated as follows.

\section{Conjecture 10.7: $\rightarrow\{$ C3 $\cup \mathrm{C} 6\}: \rightarrow[\{\mathrm{C} 1 \cap \mathrm{C} 2\} \cup\{\mathrm{C} 4 \cap \mathrm{C} 5\}]$}

A sufficient condition for a design process to progress productively towards successful culmination is the occurrence of innovation following adequate answering of the 'why' question, which in turn necessarily implies that all the determinants of a desired goal state have already been completely understood and categorically defined.

Conjecture 10.7 essentially captures the argument that we have been making in favour of a need to shift the emphasis back on the problem-creation phase of design research for intelligent systems.

Although our framework consists merely of conjectures and not formal proofs, there is a certain amount of intuitive logic in what we have proposed. Going back to our comparative model of aircraft design, we do know a lot more at this stage about the determinants of the desired goal state for flying machines than we do for intelligent machines. While there has been a proliferation of computational technologies attempting to emulate human thought and behaviour, our knowledge base of what in fact determines intelligent thought is rather inadequate. While our proposed framework might not be the best or even the only means to highlight this gap in our current state of knowledge, it does highlight a need to appropriately refocus our attention.

How can impulse-driven creativity help in finding what determines intelligent thought? It is expected that this particular science of the artificial still requires some degree of hand-holding by at least one (likely more) of the sciences of the natural as AI researchers grapple with concepts such as intelligence and consciousness and the precise physical/biological/mental processes underlying them. So the initial breakthrough (something 'absolutely new') could originate from within a contributing natural-science field such as physics or neurophysiology, which would then re-initiate the AIS design process and put it back on course towards a definite goal. We cannot, however, and do not rule out other possibilities.

\section{Discussion}

A design is something more than just a symbolic representation of an artefact for an implementer's benefit; 'it is also a small piece of theory about a part of the observable world; a micro theory, in fact, that predicts behaviour or properties that an artefact (that does not yet exist) will exhibit' (Dasgupta 1992). Dasgupta 
realises that design research in information and knowledge engineering suffers from the lack of a solid repertoire of mathematical theory such as is found in design research in mechanical or civil engineering fields.

Matters are not helped by the fact that AI researchers and systems designers seem to be confused between what we prefer to call the soft problem, that of producing some workable form of synthetic intelligence, and the primordial hard problem, that of defining the very determinants of intelligent thought. The soft problems are those that relate to specific psycho-physiological processes that go on within the brain of an intelligent biological organism. Borrowing from the natural sciences, these processes can be satisfactorily explained by applying reductionist principles (Chalmers 1995). The hard problem, however, is something intrinsically different as it does not relate to any specific neural process but rather concerns issues such as subjective experience thereby being more a question of 'why' than of 'how'. The design objective of AIS is to make it 'learn' from a given pool of knowledge by formulating a rule-based or some other form of logic architecture. While there are ways to evaluate the efficacy or otherwise of a design process in terms of how well the AIS learns and then applies this learned knowledge to accomplish a particular problem-solving task, there is, however, no way to test whether it also does acquire some subjective experience, whether it develops its own thoughts about the particular process. This limitation in the current state of design stems not from an inability to address the technological issues, which we have grouped under 'how', but rather from the unanswered 'why' question. Unlike some other engineering disciplines, information and knowledge engineering has not yet been able to fully understand the determinants of its desired goal state. Until and unless the pressing issue concerning the determinants of intelligent thought is adequately addressed, our view is that a successful culmination of the overall AIS design process is not going to happen just by continuing to pursue goal-directed design research.

We wish to be very clear, however, that by taking this stand we are not debunking or even challenging the veracity or utility of the burgeoning research in AIS and robotics and the plethora of AIS applications that seems to be touching almost all aspects of our daily lives, ranging from cars and washing machines to security systems and even to detection of dangerous diseases such as cancer. In fact, most of the state-of-the art design research that is taking place in AIS has immediate and important implications for improving the quality of our lives. That, however, simply is not the point that we have sought to raise in this chapter. We are not saying that all of the 'outputs' produced by AIS research suffer from a serious design flaw that robs them of their utility. They might be very effective in achieving their goal states. Our point is that even the most sophisticated AIS - perhaps incorporating the most powerful neural network 
or genetic algorithm or some other form of biologically inspired computational feature - is, at the end of the day, just a problem-solving device. It is in creating and defining a problem that is 'absolutely new' that the true test of AI lies - and we contend that no AIS can pass this test without fully answering the 'why'.

\section{Conclusion}

In this chapter, we have proposed a formal framework to argue in favour of the role of impulse-driven vis-a-vis goal-driven human creativity in ensuring success of the AIS design process. Our position is that the current state of design of AIS stems from a constant disregard of a key phase in the overall design process that has to ideally precede the problem-solving phase, at which, ironically, almost all current design research appears to be targeted.

Our recommended way of going about it is for AI researchers and AIS designers to think outside the MEA-inspired paradigms and look for flashes of impulsedriven creativity to first get around the perplexing 'why' question. We perceive that strong dependence on one or more established natural sciences is needed at this stage and that AI has perhaps not yet reached a state of maturity to stand on its own as an independent science. A deeper ontological inquiry into the nature of the true determinants of intelligent thought is needed before we can seek to further improve on the current state of design for artificially producing or replicating such thought.

\section{References}

Bhatta, S. R. and Goel, A. K. 1997, 'A functional theory of design patterns', Proceedings of the Fifteenth International Joint Conference on Artificial Intelligence, pp. 294-300.

Boden, M. 1990, The Creative Mind: Myths and mechanisms, Weidenfeld and Nicholson, London.

Boden, M. 1999, 'Computational models of creativity', in R. J. Sternberg (ed.), Handbook of Creativity, Cambridge University Press, UK, pp. 351-73.

Chalmers, D. J. 1995, 'Facing up to the problem of consciousness', Journal of Consciousness Studies, vol. 2, no. 3, pp. 200-19.

Colby, K. M. 1975, Artificial Paranoia: A computer simulation of paranoid processes, Pergamon Press, San Francisco. 
Cooper, R. G. and Kleinschmidt, E. J. 1995, 'Benchmarking the firm's critical success factors in new product development', Journal of Product Innovation Management, vol. 12, no. 5, pp. 374-91.

Dasgupta, S. 1992, 'Two laws of design', Intelligent Systems Engineering, vol. 1, no. 2, pp. 146-56.

Dasgupta, S. 2008, 'Shedding computational light on human creativity', Perspectives on Science, vol. 16, no. 2, pp. 121-36.

Fikes, R. E. and Nilsson, N. J. 1971, 'STRIPS: a new approach to the application of theorem proving to problem solving', Artificial Intelligence, vol. 5, no. 2, pp. 189-208.

Frantz, R. 2003, 'Artificial intelligence as a framework for understanding intuition', Journal of Economic Psychology, vol. 24, no. 2, pp. 265-77.

Gray, C. 2006, 'Flying machines', Important Thoughts About \& Experiments With Flying Machines 1485 to 1903, The Pioneer Aviation Group, Encino, Calif., viewed 25 June 2008, <http://www.flyingmachines.org>

Hearst, M. and Hirsh, H. 2000, 'AI's greatest trends and controversies', IEEE Intelligent Systems, vol. 15, no. 1, pp. 8-17.

Hevner, A. R., March, S. T., Park, J. and Ram, S. 2004, 'Design science in information systems research', MIS Quarterly, vol. 28, no. 1, pp. 75-105.

Levy, D. and Newborn, M. 1991, How Computers Play Chess, Computer Science Press, New York.

Lindsay, R., Buchanan, B. G., Feigenbaum, E. A. and Lederberg, J. 1980, Application of Artificial Intelligence for Organic Chemistry: The DENDRAL project, McGraw-Hill, New York.

McCorduck, P. 1991, Aaron's Code, W. H. Freeman \& Company, New York.

Michalos, A. C. 1970, 'Book review: The Sciences of the Artificial by Herbert A. Simon, Cambridge Mass. MIT Press 1969', Technology and Culture, vol. 11, no. 1, pp. 118-20.

Newell, A. 1982, 'The knowledge level', Artificial Intelligence, vol. 18, no. 1, pp. 87-127.

Newell, A., Shaw, J. C. and Simon, H. A. 1959, 'Report on a general problemsolving program', Proceedings of International Conference on Information Processing, pp. 256-64. 
Searle, J. R. 1980, 'Minds, brains, and programs', The Behavioral and Brain Sciences, vol. 3, pp. 417-57.

Siklossy, L. 1972, 'Natural language learning by computer', in H. A. Simon and L. Siklossy (eds), Representation and Meaning: Experiments with information processing systems, Prentice-Hall, NJ.

Simon, H. A. 1969, The Sciences of the Artificial, First edition, MIT Press, Cambridge, Mass.

Simon, H. A. 1979, 'Rational decision-making in business organizations', American Economic Review, vol. 69, pp. 495-501.

Simon, H. A. 1996, The Sciences of the Artificial, Third edition, MIT Press, Cambridge, Mass.

Stuart, R. J. and Norvig, P. 2003, Artificial Intelligence: A modern approach, Second edition, Prentice-Hall, NJ.

Turing, A. M. 1950, 'Computing machinery and intelligence', Mind, vol. 59, no. 236, pp. 433-60.

Turner, S. R. 1994, The Creative Process: A computer model of storytelling and creativity, Lawrence Erlbaum, Hillsdale, NJ.

Weizenbaum, J. 1966, 'A computer program for the study of natural language communication between man and machine', Communications of the Association of Computing Machinery, vol. 9, no. 1, pp. 36-45. 